\title{
Uncovering Individualised Treatment Effect: Evidence from Educational Trials
}

\author{
ZhiMin Xiao ${ }^{1,}{ }^{*}$, Oliver Hauser ${ }^{2}$, Charlie Kirkwood ${ }^{3}$, Daniel Z. Li ${ }^{4}$, Ben Jones ${ }^{5}$, and Steve \\ Higgins $^{6}$
}

\author{
${ }^{1}$ Graduate School of Education, University of Exeter, Exeter, EX1 2LU, UK \\ ${ }^{2}$ Department of Economics, University of Exeter, Exeter, EX4 4PU, UK \\ ${ }^{3}$ College of Engineering, Mathematics and Physical Sciences, University of Exeter, Exeter, EX4 4QF, UK \\ ${ }^{4}$ Durham University Business School, Mill Hill Lane, Durham DH1 3LB, UK \\ ${ }^{5}$ Faculty of Health, University of Plymouth, Plymouth, PL4 8AA, UK \\ ${ }^{6}$ School of Education, Durham University, Durham, DH1 1TA, UK \\ *To whom correspondence may be addressed. Email: z.m.xiao@exeter.ac.uk
}

\begin{abstract}
The use of large-scale Randomised Controlled Trials (RCTs) is fast becoming "the gold standard" of testing the causal effects of policy, social, and educational interventions. RCTs are typically evaluated - and ultimately judged — by the economic, educational, and statistical significance of the Average Treatment Effect (ATE) in the study sample. However, many interventions have heterogeneous treatment effects across different individuals, not captured by the ATE. One way to identify heterogeneous treatment effects is to conduct subgroup analyses, such as focusing on low-income Free School Meal pupils as required for projects funded by the Education Endowment Foundation (EEF) in England. These subgroup analyses, as we demonstrate in 48 EEF-funded RCTs involving over 200,000 students, are usually not standardised across studies and offer flexible degrees of freedom to researchers, potentially leading to mixed results. Here, we develop and deploy a machine-learning and regression-based framework for systematic estimation of Individualised Treatment Effect (ITE), which can show where a seemingly ineffective and uninformative intervention worked, for whom, and by how much. Our findings have implications for decision-makers in education, public health, and medical trials.
\end{abstract}

\section{Introduction}

Policy-makers interested in understanding socio-educational interventions are increasingly turning to Randomised Controlled Trials (RCTs) as a way to test the causal effects of these interventions ${ }^{1-6}$. While the use of RCTs in public policy has generally been advocated for by academic scholars (although see Deaton \& Cartwright ${ }^{7}$ and Biesta $^{8}$ for critiques and limitations of RCTs as a policy tool), the specific techniques to evaluate RCTs have received less attention. Indeed, only a fraction (less than 1\%) of Education Endowment Foundation's (EEF) £200 million budget has been allocated to the understanding and advancement of evaluation methods. Typically, RCTs are evaluated - and ultimately judged - by the Average Treatment Effect (ATE) that they achieve for the treated. The ATE captures the extent to which an intervention has had an effect on average in the study sample. Interventions that do not have a statistically significant ATE are often discarded as not meaningful and, as a result, usually not implemented more widely. However, while some interventions may not have an effect on average, they might still have a meaningful effect on a relevant subgroup of individuals, for whom the treatment is beneficial. In some cases, an intervention could thus still provide a net benefit for those individuals if rolled out. Understanding and identifying for whom a treatment works is therefore critical for policy-makers and society at large ${ }^{9}$.

One way to identify those individuals is to conduct subgroup analyses. Subgroup analyses are, of course, not new although traditional approaches usually come with several drawbacks. For instance, choosing which subgroups to analyse, and how to conduct these analyses, afford multiple degrees of freedom, potentially allowing researchers to produce and report selectively results that are supportive of their overall conclusion ${ }^{10,11}$. If this occurs, findings may be published that are not reproducible by other researchers, or they may not hold up in direct replication studies, which can shake and reduce public trust in science ${ }^{12}$. As such, subgroup analyses can sometimes be seen as "statistical malpractice" 13 , despite the obvious advantages to policy-makers if the effect of an intervention onto subgroups is better understood. In other words, subgroup analyses are as controversial as they are important: researchers "are damned if they do, and damned if they don't"13 include subgroup analyses in their research.

Building on recent insights from data science and machine learning, we propose an alternative to subgroup analyses for large-scale RCTs. As an illustration of the variation in statistical practices surrounding subgroup 
analyses, we focus on RCTs in education, which are an excellent area of research for the following reasons. First, because policy-makers are keen to improve educational practices, RCTs in educational institutions, unlike many other policy areas, have become increasingly popular ${ }^{14}$. Second, the EEF in particular, as part of their funding scheme, requires that researchers conduct a subgroup analysis of the treatment in the low-income group of Free School Meal (FSM) pupils. FSM status is one of the most frequently used variables to approximate socioeconomic status in the $\mathrm{UK}^{15,16}$.

This reporting requirement by the EEF enables us to study current practices in subgroup analyses since it is not specified (or generally agreed upon) how such analyses ought to be conceived and conducted. Previous researchers have cautioned against potential overinterpretation of effects for subgroups like FSM students ${ }^{17-20}$. Although some challenges of subgroup analyses, such as lack of statistical power and unreliable estimation, can be partially alleviated by sound analysis and responsible reporting ${ }^{17-20}$, there are insufficient statistical details in the guidance of key organisations on how to exactly estimate, report, and interpret subgroup effects ${ }^{21}$.

Our approach combines the strength of subgroup analyses, by allowing researchers to learn what works for whom, with the security of relying on a robust and replicable methodology. On the one hand, it is flexible and adaptable to a large breadth of covariates in any given RCT, allowing researchers to study Individualised Treatment Effects (ITEs) across a wide range of subgroups, particularly when they incorporate non-statistical knowledge from experts and practitioners. On the other hand, by introducing a principled procedure, it reduces researchers' degrees of freedom and ensures that findings are as robust, reproducible, and standardised as possible.

\section{Results}

\section{Conventional Approaches to Subgroup Analyses}

Following an established approach to subgroup analysis in the evaluation of EEF trials, we first employ an ordinary least squares (OLS) model, as specified in Supplementary Information (SI), with a treatment-FSM interaction term plus pre-test, a baseline measure available in almost all EEF trials. For each outcome, we obtain the sample size used for the interaction test and the $p$-value associated with the interaction term. We then deploy a multilevel model (MLM), also documented in SI, to estimate effect sizes within the subgroups of FSM and Non-FSM students using eefAnalytics ${ }^{11}$, an R package specifically developed to estimate effect sizes for RCTs of varied designs. The two subgroup effect estimates are then compared with the $p$-values from the interaction tests, which indicate if the differences between the two separately estimated subgroup effect sizes are statistically significant.

In total, we examine 84 outcomes from 48 projects (see Table S1 for details), which are largely independent projects designed and evaluated by independent teams in different years. As we have access to the raw data, this analysis differs from a standard meta-analysis. Instead, we are able to report and calculate, for each outcome (see Figures S1 to S13), three effect size estimates, their associated sample sizes and 95\% confidence intervals. The first, for reference only, is the overall effect size every EEF project reports for all the students involved. The other two are separate estimates produced by us for Non-FSM and FSM students.

Our re-analysis shows that, only 6 out of the 84 outcomes are statistically significant for FSM students, which means, consistent with the literature on overall effect sizes of EEF studies ${ }^{22}$, conventional regression analyses focusing on ATEs often produces results that are non-actionable, even when participants' lived experiences show otherwise $^{23}$. One reason, in addition to those given elsewhere ${ }^{7,22}$, this may be the case is that we conduct the same analysis across all studies, thereby holding all analyses to the same standards. Arguably, of course, our analyses following some conventional approaches to subgroup analyses are not necessarily the "correct" ones. However, this gives further credence to the need for alternatives to impact evaluation and our claim that a more consistent and replicable method to detect and calculate subgroup effects is needed.

\section{An Individualised Approach to Effect Estimation}

ATE has long been a quantity of interest in RCTs. However, "the response of the average patient to therapy is not necessarily the response of the patient being treated" 24 . Moreover, as mentioned above, current methods to identify individuals who benefited from an intervention via subgroup analyses are not always helpful.

Strong scientific interests in effect heterogeneity do exist, but detecting such variation is not always straightforward in increasingly complex designs. Many EEF trials are, for example, not even powered to detect the main effect due to challenges associated with sample size calculations ${ }^{25}$ and resource constraints. As a result, estimates of effects for FSM students usually come with the caveat, "should be interpreted with caution".

The alternative approach we propose here taps into the advancement of predictive algorithms in machine learning $^{26-29}$. It focuses on the difference in two potential outcomes ${ }^{29-31}$, often an observed factual and an 


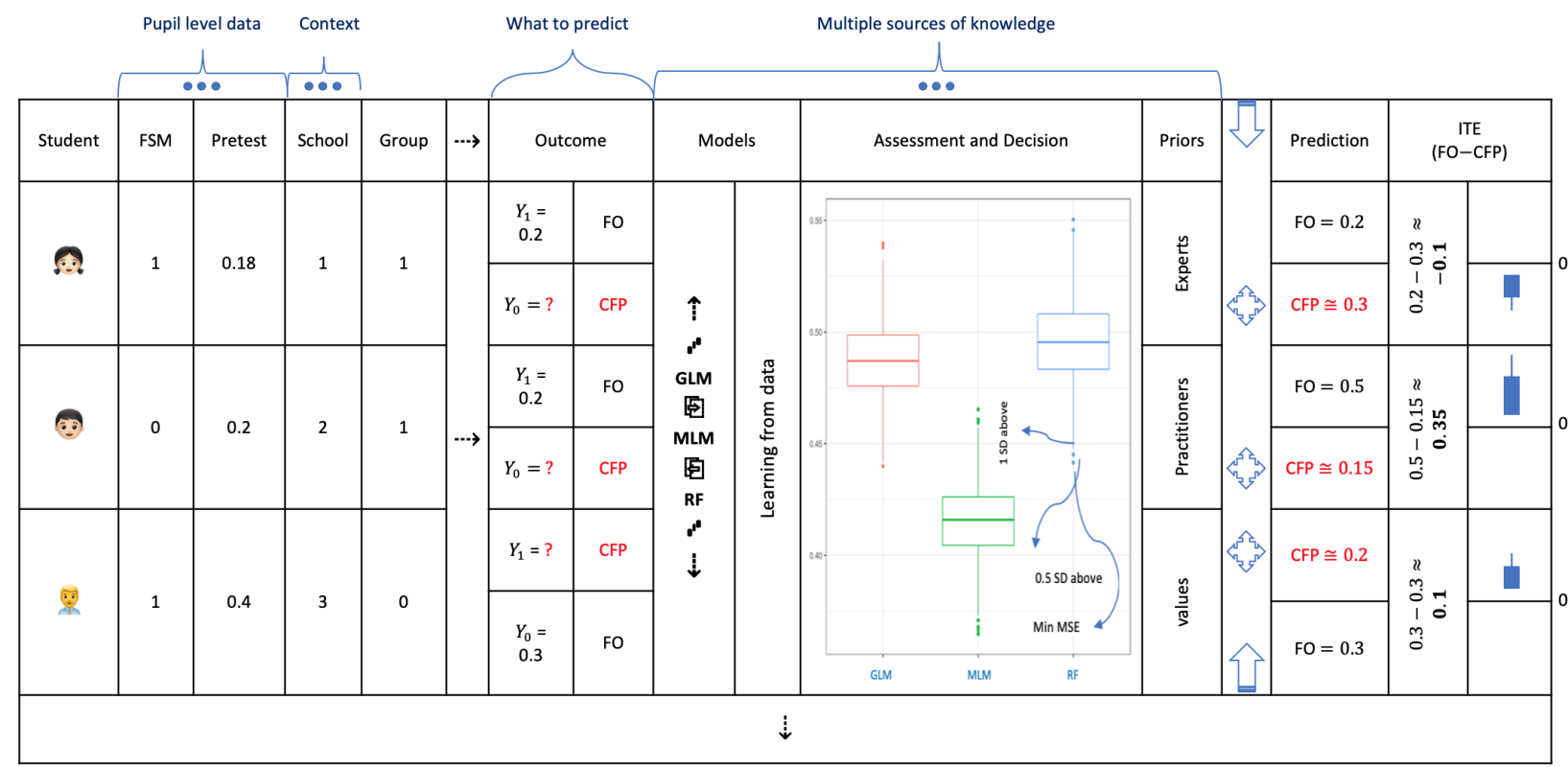

Figure 1. ITE through a graphical journey. With student- and school-level data, such as FSM status and Pretest for the former, and School ID for the latter, multiple models compete on the same data to predict counterfactual outcomes (CFP) for individual students. FO is the factual outcome observed under the treatment condition a student actually received in an intervention. The difference between FO and CFP is used to re-train the best performing model for the re-prediction of ITEs, which are then visualised as bar plots for unique individuals. Note that the ITE generation process is iterative and the arrows pointing to four directions represent the hidden procedures. There are also many more variables and even more observations in a typical policy intervention, which are visualised as dots and dotted arrows.

unobservable counterfactual outcome ${ }^{32,33}$. We call the difference Individualised Treatment Effect for a given individual in a trial that has taken place (see Figure 1 for an illustration).

When a trial is yet to be implemented, the individualised approach calculates the advantage a prospective individual is expected to have as a result of being allocated to a predicted optimal treatment arm. We call this benefit Pupil Advantage Index (PAI), given what we already know about a similar trial conducted before and the information we have about the individual to be studied ${ }^{34}$.

The evidence generated from the individualised approach is unique to specific individuals according to their observed characteristics. For each individual in an educational RCT for instance, we observe the intervention arm assigned $T \in \mathcal{T}$, the intervention outcome $Y \in \mathbb{R}$, and the student's pre-intervention and school-level characteristics, all captured by a $m$-dimensional vector $\mathbf{X} \in \mathcal{X}$. (In line with published work ${ }^{24,29,31,35}$, we use upper- and lower-case letters to denote random variables and their realised values respectively, and bold letters to represent vectors in the data.) For simplicity, we let $T=1$ denote the treatment group and $T=0$ the control or business-as-usual group, hence $T \in\{0,1\}$. Without loss of generality, we assume a higher value of $Y$ suggests a better outcome. Moreover, a deterministic decision rule $d: \mathcal{X} \rightarrow \mathcal{T}$ is a mapping from the characteristics space $\mathcal{X}$ to a treatment recommendation.

The ATE in a conventional RCT evaluation is defined as $\mathbb{E}(Y \mid T=1)-\mathbb{E}(Y \mid T=0)$. Making use of the information contained in the covariates, we define ITE as:

$$
I T E \triangleq \mathbb{E}(Y \mid \mathbf{X}, T=1)-\mathbb{E}(Y \mid \mathbf{X}, T=0) .
$$

When calculating ITE, we have an observed factual outcome, and need to predict a counterfactual outcome, had the student been assigned to an alternative intervention arm. That is to say, for a student with covariates $\mathbf{x}$ in the treatment group $T=1$, we observe the factual treatment outcome, denoted as $y_{1}(\mathbf{x})$. We utilise the observed data to predict the counterfactual outcome $\mathbb{E}(Y \mid \mathbf{X}=\mathbf{x}, T=0)$, denoted as $\hat{y}_{0}(\mathbf{x})$, and calculate the ITE as $y_{1}(\mathbf{x})-\hat{y}_{0}(\mathbf{x})$.

PAI, nevertheless, is computed prospectively, and it is a predicted net benefit when a student is assigned to an optimal intervention arm. For instance, if we predict that assigning a student to the control group generates a higher outcome, then PAI is equal to the predicted control outcome minus the predicted treatment outcome. Therefore, 
PAI is non-negative and equal to the absolute value of ITE. Put formally,

$$
P A I \triangleq|\mathbb{E}(Y \mid \mathbf{X}, T=1)-\mathbb{E}(Y \mid \mathbf{X}, T=0)| .
$$

When computing PAI, we are yet to assign a student to an intervention arm, but we do have some knowledge, namely, the observed covariates $\mathbf{x}$, about the students to be studied. As before, we denote the predicted potential post-test outcomes by $\hat{y}_{1}(\mathbf{x})$ and $\hat{y}_{0}(\mathbf{x})$ respectively, where the subscript represents the intervention arm assigned. Supposing a student should be optimally assigned to the control arm, in the sense that $\hat{y}_{0}(\mathbf{x}) \geq \hat{y}_{1}(\mathbf{x})$, the corresponding PAI, by definition, is thus $\hat{y}_{0}(\mathbf{x})-\hat{y}_{1}(\mathbf{x})$.

In other words, the ITE can be positive, zero, or negative, whereas the PAI is expected to be positive, unless a trial aims to minimise a certain risk ${ }^{36}$. This is because the PAI is a predicted advantage score if the person were to be allocated to an optimal treatment arm, either treatment or control (assuming, for simplicity, that there are only two treatment options). The PAI is a quantitative estimate of the magnitude by which the optimal treatment is predicted to outperform an active alternative or control ${ }^{34}$. That is, the PAI might suggest that, for the given individual, not intervening (i.e., keeping them in the control) may be the optimal choice, or the allocation of treatment options may embrace individual preferences and take into consideration other factors such as $\operatorname{cost}^{34}$, local knowledge, and/or professional judgement. Put differently, the PAI is best described as individualised treatment recommendation ${ }^{34}$, which can be useful to decision-makers when they have a number of options to choose from for a given individual.

A number of models can be deployed to predict potential outcomes. Suppose we have a pre-specified model $f(\mathbf{x} ; T)$ for the population approximation of the intervention outcome, an optimal decision rule, $d^{*}(\cdot)$, is defined as follows

$$
d^{*}(\mathbf{x} ; f) \triangleq \underset{T \in \mathcal{T}}{\arg \max } f(\mathbf{x}, T) .
$$

That is, given the model $f$ and the observed covariates $\mathbf{x}, d^{*}$ specifies an optimal intervention for the student. Note that the prediction model $f$ is an approximation, as the true data generation process for the student $i$ is

$$
Y_{i}=\underbrace{f\left(\mathbf{X}_{i}, T_{i}\right)+\xi_{i}\left(\mathbf{X}_{i}, \mathbf{U}_{i}, T_{i}\right)}_{\mathbb{E}\left[Y_{i} \mid \mathbf{X}_{i}, \mathbf{U}_{i}, T_{i}\right]}+\varepsilon_{i}
$$

where the first two terms represent the true conditional expectation and the last term is the irreducible error around it $^{24}$. Unfortunately, the true data generation process is unknown, even in the absence of $\varepsilon_{i}$, as $f$ will always differ from the true process by $\xi_{i}\left(\mathbf{X}_{i}, \mathbf{U}_{i}, T_{i}\right)$, where $\mathbf{U}_{i} \in \mathcal{U}$ represents unobserved and unobservable covariates, such as culture, tradition, and value-based decisions. That is, we cannot gather data on all possible covariates in a study, and we also need to recognise that researchers bring different assumptions into the data generation process at collection, pre-processing, and analysis stages ${ }^{37}$. Yet, given sufficient covariates, these models can yield insightful information about the ITE and/or the PAI.

As an illustration of the individualised approach to subgroup analyses, we focus on one EFF-funded dataset from a trial called Chess in Schools ${ }^{23}$. This project had 100 schools randomly assigned to either intervention or control, involving 4,009 pupils. Intervention schools taught children how to play chess over a year, whereas control schools were business-as-usual. The primary outcome was Key Stage 2 maths score - an important standardised test taken by UK pupils usually at age 11 - one year after the intervention.

We highlight the above project for the following reasons. First, it has a relatively large sample size and the highest possible security rating of five padlocks, which indicates high internal and external validity ${ }^{38}$. Second, at a total cost of $£ 689,150$, the project was a large-scale and intensive effort to improve math skills, yet the reported overall effect size of $0.01(-0.15,0.16)^{23}$ might suggest that it was not a worthwhile investment, prompting the question whether this intervention did have some non-negligible and educationally meaningful effects for some students, given the report that $50 \%$ of the pupils said "they liked the chess lessons a lot" and "teachers were very positive about the intervention and its impact on pupils' skills and behaviour" 23 . Finally, the dataset has many observed covariates, which makes it suitable for this individualised approach. Any other RCT that fulfils the above criteria would make a suitable candidate for this approach.

Using generalised linear model (GLM), multilevel model (MLM), and random forests (RF) as model candidates, we make a chain of predictions. For each individual, we observe one treatment condition allocated and an outcome for each individual, which we call Factual Outcome (FO). But to assess model performance, we need to predict an outcome for each individual and compare it with the observed FO. Let's call the predicted outcome for the condition a student actually receives Factual Prediction (FP), and the predicted outcome for the condition the student does 
not receive Counter-Factual Prediction (CFP). Each predictive model generates a factual and a counterfactual prediction for each and every student, and the difference between the two predicted outcomes (FP and CFP), or between FO and CFP, is our ultimate quantity of interest.

We follow an established practice of bootstrapping in machine learning to conduct model selection. Amongst the 1,000 sets of parameters as a result of random sampling for training and testing, one set of each model has the lowest average out-of-sample prediction error (MSE), which should be used to make factual and counterfactual predictions. However, to minimise bias in the prediction ${ }^{39}$, we also take into consideration the variation in prediction and use two other sets of parameters from each model candidate (see Table S2 and SI for details).

When the target of prediction is a potential outcome (CFP), as plotted in Figure S14, the two linear models perform better than the RF. Nevertheless, when the individualised effect is calculated as the difference between two predicted potential outcomes (i.e., FP - CFP), GLM and MLM (but not RF) produce a constant effect equal to the corresponding regression coefficient on treatment indicator of the linear model under the chosen set of parameters (see Table S2). Note that individualised effects, when calculated as FO - CFP, are not constant, regardless of the candidate model, but those from linear models have greater variations than those from the RF, as shown in Figure 2 (A). For the above reasons, we choose RF, a candidate model yielding results with a variation between two extremes (constant or too wide), and re-train it for the final target of prediction, namely, ITEs (see SI for details).
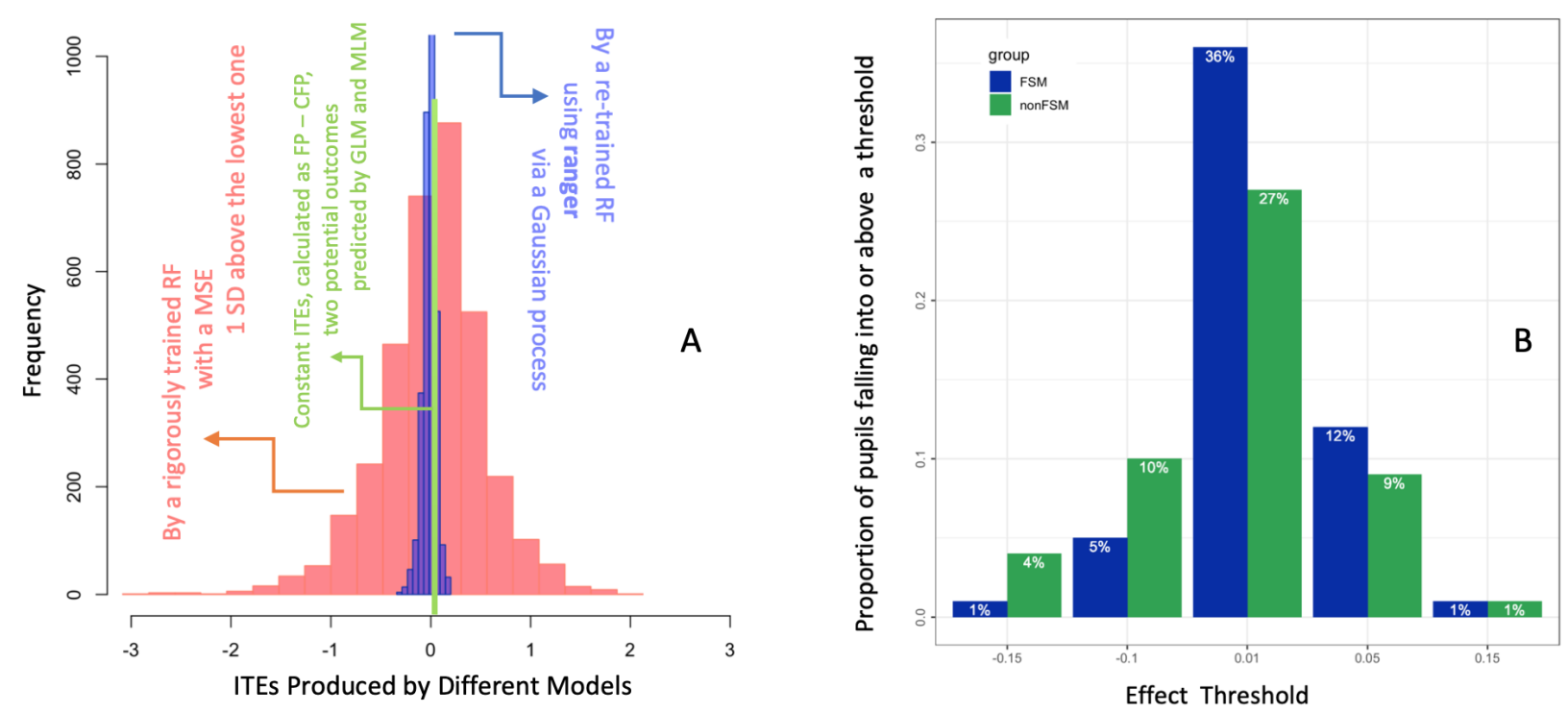

Figure 2. ITEs for use. A: Constant ITEs at 0.01, calculated as FP - CFP, two potential outcomes predicted by the two linear candidate models. Histograms of two distributions of ITEs from two types of RF, one predicting one potential outcome and the other re-trained to predict the differences of two, i.e., ITEs. B: ITE thresholds used to visualise the proportions of FSM and Non-FSM students predicted to benefit (if positive) or lose (if negative) from the intervention by at least a chosen amount.

With ITEs from the re-trained RF, we visualise in Figure 2 (B) the proportions of FSM and Non-FSM students at different ITE thresholds, which are set according to what we know from the literature and understand about the trial under investigation. For instance, either the overall effect size or that for FSM students reported by the original evaluation team is $0.01^{23}$, and the average of all the EEF trials funded to date is about $0.05^{22}$. Other values in the figure reflect the distribution of our predicted ITEs. We find that $36 \%$ of the FSM students in the study benefited at least $0.01 s d$ from the Chess intervention, whereas $27 \%$ of the Non-FSM students reaped the same amount of benefit from the trial. The pattern continues, such that more FSM students benefit from the intervention than Non-FSM students, up to $0.05 s d$. A small fraction, equally made up of FSM and non-FSM students, benefits at $0.15 s d$. On the negative side, fewer FSM students were worse off than their Non-FSM counterparts by at least 0.1 or $0.15 s d$. Taken together, our analysis reveals that the intervention was more beneficial to FSM students than to Non-FSM students, amplifying the positive feedback given by the pupils and teachers actually involved in the trial ${ }^{23}$. 


\section{Discussion}

In line with previous research, we proposed an individualised approach here to effect estimation, which employed three types of predictive algorithms to first predict how individuals would respond to different treatment options. We then quantified the differences in potential outcomes at individual level, which were subsequently used to re-train the best of the three candidate models to predict the ultimate quantity of interest, namely, ITEs for unique individuals. These highly individualised effect estimations can be utilised to evaluate an existing intervention or optimise treatment recommendations for individuals in a trial that is yet to be implemented.

The ITEs can be grouped by FSM status, a variable used for subgroup analyses. Choosing this grouping variable also relates to the policy problem we started with and, while we focus on ITEs, it is still important to see if and how they converge with the ATE when aggregated, as schools and policy-makers in education often need to consider students in groups, be they classes, year groups, schools, or districts. We demonstrated that the results from the individualised approach could reproduce the original ATE and answer policy-relevant questions about subgroups of students, such as the most disadvantaged students.

In sum, we have shown that, while conventional approaches to effect size estimation in policy interventions often resort to aggregated measures of impact, such as ATE or conditional $\mathrm{ATE}^{35,40}$, these evaluations take little note of individual characteristics that may alter individual responses to interventions. After all, an intervention that worked well on average may not be the best option for all ${ }^{41}$. Any subgroup analysis, or heterogeneous treatment effects analysis, is one step closer towards a more sensitive estimation of individual responses to an intervention ${ }^{42}$, but current practices are not standardised. Heterogeneous causal effect estimation using causal forests permits statistical inferences and quantifies their uncertainties, thanks to its asymptotic properties associated with a normality theory ${ }^{31,35}$. Nevertheless, it is worth pointing out that no method can lead to a truly individualised effect ${ }^{43,44}$ : no individual can ever be in both the control and the treatment condition. However, the methods proposed here and elsewhere by others ${ }^{29}$ can get closer to an individualised estimation. Future research will continue to develop consistent and efficient ways to get these estimates, as the current process is often "data hungry" with half-half sampling split for feature space construction and effect estimation. As Wager \& Athey note, however, the sampling split rule is arbitrary and "still in its infancy" 31.

As in any research, our individualised approach has its own limitations. First, while it focuses on evidence that is actionable, the prediction procedures could have been more dynamic (i.e., each individual may have her/his own best prediction algorithm, rather than a best performing algorithm for all) and the uncertainties surrounding those ITEs are yet to be formalised and refined. Second, we could have simulated some data from an RCT where the intervention effect is zero and no relationships exist amongst covariates. If the predictive algorithms fail to identify individuals who benefit from the "trial", we would be much more confident about the approach. It would be even better if we can test the approach in multi-phase trials and/or deploy it to predict outcomes in longitudinal studies.

Finally, while our approach has shown to work effectively in the education sector, it is worth pointing out that it can be applicable and relevant in any area of science and policy - from tax collection to medical trials to public health. The importance of individualising treatment effects is particularly critical in areas of rapid development and testing of drugs or vaccines, or of policies that encourage certain behaviours for the public good. Take, for example, the COVID-19 crisis: first, vaccine trials might benefit from the ITE approach, in that even a vaccine that only works for a relevant subgroup (e.g., patients with underlying health conditions) would be a much-needed advancement to battle this deadly disease; second, encouraging social distancing may take different forms and policy-makers would be well-advised to understand how different subgroups (e.g., the elderly, young people, and key workers) might respond to different messaging.

\section{Methods Summary}

For the demonstration, the Chess dataset we construct has 16 variables, including the aforementioned outcome measure and treatment indicator. Pre-treatment covariates consist of Key Stage 1 measures and pupil-level characteristics such as FSM status. To be consistent with the way most EEF evaluators deal with missing data in their primary analyses, we also remove all the rows with missing data, which is less problematic to machine learning algorithms such as random forests than it is to inferences based on probability theories ${ }^{45}$. We end up with a sample of 3,514 complete cases, which is unsurprisingly smaller than 3,865 the evaluation team reported and 3,695 for the interaction test in Figure S1, as the variables used to construct the data are different.

To conduct model selection, we first randomly split, via bootstrapping, the observed RCT data into two disjoint training and testing subsets. The training set is used to train candidate models, and the testing set to assess their performances. In each bootstrapped re-sample, an outcome of interest is generated by each model and for each 
student. An average error, namely mean squared error (MSE), in prediction across all the individuals in the testing set is produced. This process is repeated 1,000 times to generate a distribution of the MSEs for each model. We then choose one type of model, taking into consideration its variation in prediction, that has the best performance on average in the testing set for the final prediction of ITEs. Note that the highlighted word "best" here and elsewhere in the paper does not suggest the best of all possible models, it only means the best amongst the models deployed, i.e., GLM, MLM, and RF. The GLM for prediction here differs from the earlier OLS model for interaction test, as the former has more variables than the latter in order to predict well and is less concerned about collinearity and

the coefficient of a particular variable. GLM and MLM are the primary evaluation models in almost all EEF trials, it is natural to compare them with RF, which have excellent performances in prediction ${ }^{31,35,39,43,46}$.

\section{References}

1. Banerjee, A. et al. A multifaceted program causes lasting progress for the very poor: Evidence from six countries. Science 348, DOI: 10.1126/science.1260799 (2015).

2. Dillon, M. R., Kannan, H., Dean, J. T., Spelke, E. S. \& Duflo, E. Cognitive science in the field: A preschool intervention durably enhances intuitive but not formal mathematics. Science 357, 47-55, DOI: 10.1126/science. aal4724 (2017).

3. Higgins, S. Improving Learning: Meta-analysis of Intervention Research in Education (Cambridge University Press, Cambridge, 2018).

4. Rogers, T. \& Feller, A. Reducing student absences at scale by targeting parents' misbeliefs. Nat. Hum. Behav. 2, 335-342, DOI: 10.1038/s41562-018-0328-1 (2018).

5. Thaler, R. H. \& Sunstein, C. R. Nudge: Improving Decisions About Health, Wealth, and Happiness (Yale University Press, New Haven; London, 2008).

6. John, P. et al. Nudge, Nudge, Think, Think: Experimenting with Ways to Change Civic Behaviour (Bloomsbury Academic, London, 2011).

7. Deaton, A. \& Cartwright, N. Understanding and misunderstanding randomized controlled trials. Soc. Sci. Medicine 210, 2-21, DOI: 10.1016/j.socscimed.2017.12.005 (2018).

8. Biesta, G. J. J. Why 'What Works' Still Won't Work: From Evidence-Based Education to Value-Based Education. Stud. Philos. Educ. 29, 491-503, DOI: 10.1007/s11217-010-9191-x (2010).

9. Athey, S. Beyond prediction: Using big data for policy problems. Science 355, 483-485, DOI: 10.1126/science. aal4321 (2017).

10. Simmons, J. P., Nelson, L. D. \& Simonsohn, U. False-positive psychology: undisclosed flexibility in data collection and analysis allows presenting anything as significant. Psychol. Sci. 22, 1359-1366, DOI: 10.1177/ $0956797611417632(2011)$.

11. Xiao, Z., Kasim, A. \& Higgins, S. Same difference? Understanding variation in the estimation of effect sizes from educational trials. Int. J. Educ. Res. 77, 1-14, DOI: 10.1016/j.ijer.2016.02.001 (2016).

12. Wingen, T., Berkessel, J. B. \& Englich, B. No Replication, No Trust? How Low Replicability Influences Trust in Psychology. Soc. Psychol. Pers. Sci. DOI: 10.1177/1948550619877412 (2019).

13. Petticrew, M. et al. Damned if you do, damned if you don't: subgroup analysis and equity. J. Epidemiol. \& Community Heal. 66, 95-98, DOI: 10.1136/jech.2010.121095 (2012).

14. Connolly, P., Keenan, C. \& Urbanska, K. The trials of evidence-based practice in education: a systematic review of randomised controlled trials in education research 1980-2016. Educ. Res. 60, 276-291, DOI: 10.1080/ 00131881.2018 .1493353 (2018).

15. Hobbs, G. \& Vignoles, A. Is children's free school meal 'eligibility' a good proxy for family income? Br. Educ. Res. J. 36, 673-690, DOI: 10.1080/01411920903083111 (2010).

16. Strand, S. School effects and ethnic, gender and socio-economic gaps in educational achievement at age 11. Oxf. Rev. Educ. 40, 223-245, DOI: 10.1080/03054985.2014.891980 (2014).

17. Assmann, S. F., Pocock, S. J., Enos, L. E. \& Kasten, L. E. Subgroup analysis and other (mis)uses of baseline data in clinical trials. The Lancet 355, 1064-1069, DOI: 10.1016/S0140-6736(00)02039-0 (2000).

18. Lagakos, S. W. The challenge of subgroup analyses - Reporting without distorting. New Engl. J. Medicine 354, 1667-1669, DOI: 10.1056/NEJMp068070 (2006). 
19. Song, F. \& Bachmann, M. Cumulative subgroup analysis to reduce waste. BMC Medicine 14, 1-8, DOI: 10.1186/s12916-016-0744-x (2016).

20. Wang, R., Lagakos, S. W., Ware, J. H., Hunter, D. J. \& Drazen, J. M. Statistics in Medicine: Reporting of Subgroup Analyses in Clinical Trials. New Engl. J. Medicine 357, 2189-2194, DOI: 10.1056/NEJMsr077003 (2007).

21. Wijn, S. R. W. et al. Guidance from key organisations on exploring, confirming and interpreting subgroup effects of medical treatments: a scoping review. BMJ Open 9, e028751, DOI: 10.1136/bmjopen-2018-028751 (2019).

22. Lortie-Forgues, H. \& Inglis, M. Rigorous Large-Scale Educational RCTs Are Often Uninformative: Should We Be Concerned? Educ. Res. 48, 158-166, DOI: 10.3102/0013189X19832850 (2019).

23. Jerrim, J., Macmillan, L., Micklewright, J., Sawtell, M. \& Wiggins, M. Chess in Schools. Tech. Rep., London (2016).

24. Kapelner, A. et al. Inference for the Effectiveness of Personalized Medicine with Software. arXiv (2014). 1404.7844

25. Schulz, K. F. \& Grimes, D. A. Sample size calculations in randomised trials: Mandatory and mystical. Lancet 365, 1348-1353, DOI: 10.1016/S0140-6736(05)61034-3 (2005).

26. Breiman, L. Statistical Modeling: The Two Cultures. Stat. Sci. 16, 199-231, DOI: 10.1214/ss/1009213726 (2001).

27. James, G., Witten, D., Hastie, T. \& Tibshirani, R. An Introduction to Statistical Learning: with Applications in $R$ (Springer, New York, 2013), 6th edn.

28. van der Laan, M. J. \& Rose, S. Targeted learning in data science: causal inference for complex longitudinal studies (Springer, 2018).

29. Alaa, A. M. \& van der Schaar, M. Validating causal inference models via influence functions. In 36th International Conference on Machine Learning, ICML 2019 (Long Beach, California, 2019).

30. Rubin D. B. Estimating causal effects of treatment in randomized and nonrandomized studies. J. Educ. Psychol. 66, 688-701 (1994).

31. Wager, S. \& Athey, S. Estimation and Inference of Heterogeneous Treatment Effects using Random Forests. $J$. Am. Stat. Assoc. 113, 1228-1242, DOI: 10.1080/01621459.2017.1319839 (2018).

32. Hernán, M. A., Hsu, J. \& Healy, B. A Second Chance to Get Causal Inference Right: A Classification of Data Science Tasks. Chance 32, 42-49, DOI: 10.1080/09332480.2019.1579578 (2019).

33. Pearl, J. \& Mackenzie, D. The Book of Why: The New Science of Cause and Effect (Penguin, 2019).

34. DeRubeis, R. J. et al. The personalized advantage index: Translating research on prediction into individualized treatment recommendations. A demonstration. PLoS ONE 9, 1-8, DOI: 10.1371/journal.pone.0083875 (2014).

35. Athey, S. \& Imbens, G. Recursive partitioning for heterogeneous causal effects. Proc. Natl. Acad. Sci. United States Am. 113, 7353-7360, DOI: 10.1073/pnas.1510489113 (2016).

36. Manski, C. F. Treatment Choice With Trial Data: Statistical Decision Theory Should Supplant Hypothesis Testing. Am. Stat. 73, 296-304, DOI: 10.1080/00031305.2018.1513377 (2019).

37. Meng, X.-L. Dissecting multiple imputation from a multi-phase inference perspective: What happens when God's, imputer's and analyst's models are uncongenial? Stat. Sinica 27, 1485-1594, DOI: 10.5705/ss.2014.067 (2017).

38. Higgins, S. et al. The Sutton Trust-Education Endowment Foundation Teaching and Learning Toolkit. Tech. Rep., Education Endowment Foundation, London (2015).

39. Athey, S. \& Imbens, G. W. Machine Learning Methods That Economists Should Know About. Annu. Rev. Econ. 11, 685-725, DOI: 10.1146/annurev-economics-080217-053433 (2019).

40. Lamont, A. et al. Identification of predicted individual treatment effects in randomized clinical trials. Stat. Methods Med. Res. 0, 1-19, DOI: 10.1177/0962280215623981 (2016).

41. Schnell, P. M., Tang, Q., Offen, W. W. \& Carlin, B. P. A Bayesian credible subgroups approach to identifying patient subgroups with positive treatment effects. Biometrics 72, 1026-1036, DOI: 10.1111/biom.12522 (2016). 
42. Seibold, H., Zeileis, A. \& Hothorn, T. Individual treatment effect prediction for amyotrophic lateral sclerosis patients. Stat. Methods Med. Res. 0, 1-22, DOI: 10.1177/0962280217693034 (2017).

43. Athey, S. \& Imbens, G. W. The state of applied econometrics: Causality and policy evaluation. J. Econ. Perspectives 31, 3-32, DOI: 10.1257/jep.31.2.3 (2017).

44. Alaa, A. M. \& van der Schaar, M. Limits of estimating heterogeneous treatment effects: Guidelines for practical algorithm design. In 35th International Conference on Machine Learning, ICML 2018 (Stockholm, Sweden, 2018).

45. Shmueli, G. To explain or to predict? Stat. Sci. 25, 289-310, DOI: 10.1214/10-STS330 (2010).

46. Xiao, Z. \& Higgins, S. The Power of Noise and the Art of Prediction. Int. J. Educ. Res. 87, 36-46, DOI: 10.1016/j.ijer.2017.10.006 (2018).

\section{Author contributions statement}

Z.M.X. conceived the project, conducted the analyses, produced most of the figures, and wrote many drafts. O.H. and Z.M.X. refined the individualised treatment effect concept, the figures, and the main findings through numerous meetings and discussions. C.K. and B.J. contributed to the analyses involving machine learning and regression techniques respectively. D.Z.L. improved the mathematical underpinnings of ITE and PAI. S.H. secured access to the data archive, posed the original question about the value of a treatment-FSM interaction test, and expressed an interest in understanding the distribution of effects. All authors reviewed multiple drafts of the manuscript. 